\title{
Low-Noise Flapping Wings with Tensed Membrane
}

\author{
Marco Debiasi ${ }^{1 \S}$ \\ Centre for Defence Engineering, Cranfield University, Shrivenham, SN6 8LA, United Kingdom \\ Zhenbo Lu, ${ }^{2}$ Quoc Viet Nguyen, ${ }^{3}$ and Woei Leong Chan ${ }^{4}$ \\ Temasek Laboratories, National University of Singapore, 117411, Singapore \\ ${ }^{\S}$ Corresponding author: marco.debiasi@cranfield.ac.uk; (+44) 1793785375
}

\begin{abstract}
Micro air vehicles with flapping wings have the potential to be both more efficient and manoeuvrable than similar-sized fixed or rotary-wing aircraft. Reducing the perceived noise produced by flapping wings without compromising or possibly enhancing their aerodynamic performance would be crucial in surveillance and military applications. To this aim we have designed and fabricated flapping wings whose supporting stiffeners tense the Mylar membrane to which they are bonded. The sound produced by these wings at different flapping frequencies has been recorded in an anechoic chamber simultaneously to the upward thrust they create. Comparing their characteristics and performance with those of flapping wings of conventional design suggests that tensing the wing's membrane can increase the thrust while decreasing the typical rustling noise of the flapping wings.
\end{abstract}

\section{Nomenclature}

$\begin{array}{ll}f & =\text { frequency, } \mathrm{Hz} \\ F_{z} & =\text { upward thrust, } \mathrm{g} \\ p^{\prime} & =\text { pressure fluctuation, } \mathrm{Pa} \\ p_{\text {ref }} & =\text { reference pressure, } \mu \mathrm{Pa} \\ t & =\text { time, } \mathrm{s}\end{array}$

\footnotetext{
${ }^{1}$ Senior Research Scientist, Temasek Laboratories, National University of Singapore; currently Research Fellow, Cranfield University, United Kingdom, Member AIAA.

${ }^{2}$ Senior Research Scientist, Temasek Laboratories, National University of Singapore.

${ }^{3}$ Senior Research Scientist, Temasek Laboratories, National University of Singapore.

${ }^{4}$ Senior Research Scientist, Temasek Laboratories, National University of Singapore.
} 


$$
\begin{array}{ll}
T & =\text { flapping-cycle period, } \mathrm{s} \\
\phi & =\text { flapping angle } \\
\Phi & =\text { flapping-angle amplitude }
\end{array}
$$

\section{Introduction}

IICRO air vehicles (MAVs) have the potential to revolutionize the sensing and information gathering capabilities in both military and civil fields. Fixed wing, rotary wing, and flapping wing (FW) are the three main vehicle concepts [1-6], the latter having very attractive characteristics for flight inside confined spaces, such as high maneuverability and high efficiency at very-low speed, vertical take-off, hovering or nearly hovering. Well known FW-MAVs are the DelFly [7, 9] from TU Delft and the RoboBee [10, 12] from Harvard University.

The wings of current FW-MAVs resemble those of insects and bats which can provide inspiration for optimizing their aerodynamic and acoustic characteristics. Insect wings are one of nature's lightest structures; lacking bones and muscles, these wings cannot actively change their shape since they consists of a thin panel with stiffening veins of chitin, a tough material that also composes an insect's hard outer skin [13]. Insect wings have complex aerodynamics as they produce highly unsteady flow in a range of very low Reynolds numbers $[1,2,14,15]$. This makes it difficult to mathematically quantify their characteristics and to calculate their lift. Several experimental [16-23] and numerical [24-29] studies have been conducted to understand the aerodynamics of insect wings. Conversely, fewer studies exist on the sound produced by flying insects [30-34], albeit its acoustic features and generation mechanisms are relevant to the basic knowledge of insect physiology and evolution [35] and to insects' biomimetic technologies. The bumbling or buzzing of bees or mosquitoes are well-known sounds produced by their flapping wings. The sound is generated by the interaction of the flow features (such as leading edge vortex, wing tip vortex, and trailing edge vortex) around the wings [17-20, 22].

Bat's membrane wings are mechanically the most sophisticated of animal wings. They consist of a highly evolved and specialized arm/hand structure that allows a high degree of control of the shape and tension of the skin membrane (patagium) it supports. This allows rapid active control of the aerodynamic and acoustic performance of their wings in a wide range of flight conditions $[36,37]$.

Inspired by these, the current work explored two design variations of the standard wings of the FlowerFly, a FWMAV developed by the NUS Temasek Laboratories whose wings are fabricated using thin sheets of Mylar for their 
membrane and carbon-fiber rods of different diameters for their supporting stiffeners [38]. Wings of this type typically create a characteristic and undesirable rustling sound when flapping, possibly a result of the complex flexing and twisting of the Mylar membrane, a strong and stiff material that can bend only because manufactured in very thin sheets. In a previous study the authors explored the perceive-noise benefit of using different softer materials for the fabrication of the wing's membrane of a two-winged FW-MAV [39]. This study explores a different noise-reducing approach applied to a more practical FW-MAV configuration where by slightly bending some of the rods a tension can be induced in some portions of the membrane which can be used to tailor its aerodynamic and its acoustic behavior. This in some ways emulates a bat's wing albeit in a passive rather than active way. Simultaneous measurements of the noise and of the upward thrust produced by the wings were performed in an anechoic chamber to identify the wing configuration with lower perceived noise per unit thrust. The wings were mounted on the flapping mechanism of the FlowerFly and were operating at flapping frequencies from 8 to $13 \mathrm{~Hz}$ which are representative of the flight conditions of this FW-MAV.

\section{Experimental Setup}

\section{A. Flapping-wing Mechanism}

The flapping-wing mechanism used in this project is the one of the FlowerFly which is described in more detail in Ref. [38]. It suffices here to recall that it consists of a gear box which synchronously drives four wings and creates double clap-and-fling effects during one flapping cycle.

The gear-box design, Fig. 1, combines two modules driven by a single motor placed at the center of the gear box. Each module consists of a crank-slider and linkage (two couplers and two output links), and a pair of wings. The flapping angle $\phi$ of each output link to which the wing is attached was designed to have an amplitude $\Phi$ of $90^{\circ}$ and is plotted in Fig. 1. Accordingly, the flapping angle of the flapping-wing system is $360^{\circ}$ for a flapping cycle, Fig. 2, and the clap-and-fling of the wings is created at the end of each half flapping stroke: downstroke and upstroke. The

large flapping angle and clap-and-fling effect help to produce high thrust. A gear ratio of 1:20 has been chosen for the gear box assembly to reduce the motor speed and produce a high driving torque at the output links. 


\section{B. Flapping Wings}

Figure 3 shows couples of tested wings. Two couples of each type (4 wings in total) were installed in the flapping mechanism. Wings A are the standard (reference) type used in the FlowerFly at the time of this study. Their membrane consists of a $10 \mu \mathrm{m}$-thick Mylar sheet supported by carbon-fiber rods of diameter $0.8 \mathrm{~mm}$ at the leading edge and $0.6 \mathrm{~mm}$ at the wing root. The latter rods are angled $5^{\circ}$ inward toward the trailing edge such that once mounted straight in the flapping mechanism they create a slack of the membrane close to the wing root. This allows alternate passive tilt of the wings in the downstroke and upstroke phases of a flapping cycle. Smaller $0.3 \mathrm{~mm}$ diameter rods are used as stiffeners of the wing membrane. The weight of a single wing A is $0.346 \mathrm{~g}$.

Wings A1 are similar to the A type except that their wing-root rods are not angled. This reduces the slack of the membrane close to the wing root thus making the membrane more tensed during flapping which is thought to reduce the corresponding noise. The weight of a single wing A1 is $0.350 \mathrm{~g}$. Wings B have a $5 \mu \mathrm{m}$-thick Mylar sheet membrane supported by carbon-fiber rods of diameter $0.7 \mathrm{~mm}$ at the leading edge and $0.5 \mathrm{~mm}$ at the wing root in the same arrangement of wing A. However the arrangement of the $0.3 \mathrm{~mm}$ rods is very different and comprises a curved rod that flexes around the leading edge, the tip, and part of the trailing edge. This introduces tension in most of the wing membrane except close to the wing root. This tension is hoped to further reduce the flapping noise. A diagonal stiffener is also added to reduce wing flexibility when the wing rotates. This stiffener stores energy at the start of a stroke, and releases energy at the end of the stroke to ensure faster and smaller wing fluctuation on its rotation. The weight of a single wing $\mathrm{B}$ is $0.406 \mathrm{~g}$.

\section{Acoustic and Upward Thrust Measurements}

Simultaneous acoustic and thrust measurements of the FlowerFly flapping wings in a range of frequencies representative of its flight conditions were performed inside the acoustic anechoic chamber of the NUS Temasek Laboratories. The inner walls of this chamber, originally designed for measurements of jet noise, are covered with polyurethane-foam acoustic wedges (Illbruck SONEXsuper) with an absorption coefficient higher than 1.0 for frequencies above $500 \mathrm{~Hz}$. The inner dimensions between the wedges are about $2 \mathrm{~m} \times 2 \mathrm{~m} \times 2 \mathrm{~m}$.

The flapping wing models were installed at the center of the chamber as shown in Fig. 4. They were mounted on a supporting post firmly fixed to the chamber's ceiling for suppressing any vibration generated by the flapping model. This overhanging mount configuration prevents the supporting post to interfere with the downward flow created by the flapping wings. The noise produced by the flapping wings was recorded by two $1 / 2$ inch condenser 
microphones installed at the same height of the flapping wings on a rotatable frame from the floor that allows positioning them at different angles around the model, Fig. 4. A servo motor rotated the frame with the microphones around the model. Measurements were taken at $45^{\circ}$-spaced angles as indicated in Fig. 4 . The radial distance between the tips of the flapping wings and the tips of the microphones is about $500 \mathrm{~mm}$.

The microphones (Brüel \& Kjær Models 4953) have a flat frequency response up to $15 \mathrm{kHz}$ and thus are suitable to measure noise in the range audible by humans. The microphones were connected to preamplifiers and a signal conditioner (Brüel \& Kjær Models 2669 and NEXUS 2690-A, respectively). Their signal was sampled at $50 \mathrm{kHz}$ by a fast analog-to-digital board (National Instruments PXI 6221) installed in National Instruments PXIe-8133 embedded controller. Each recording consists of $5 \times 10^{5}$ samples (thus the length of the signal timetrace is $10 \mathrm{~s}$ ). The signal was low-pass filtered at $24,899 \mathrm{~Hz}$ by a Butterworth filter to avoid aliasing. The narrowband power spectrogram of the microphone voltage was computed using a 2048-point short-time Fourier transform, which provided a spectral resolution of about $24 \mathrm{~Hz}$. Using each microphone's sensitivity (about $48 \mathrm{mV} / \mathrm{Pa}$ ) and accounting for the amplifier gain setting, the voltage power spectrogram was converted to the power spectrogram of $p^{\prime} / p_{r e f}$, where $p^{\prime}$ is the pressure fluctuation and $p_{\text {ref }}=20 \mu \mathrm{Pa}$ is the commonly used reference pressure. Converted to decibels, this becomes the spectrogram of the sound pressure level, $\operatorname{SPL}(f)$, where $f$ is the measured frequency. Aweighting correction was applied to the SPL spectrogram to account for the relative loudness perceived by the human ear.

Time integration of the spectrogram values provides the corresponding SPL spectrum. The overall sound pressure level is then obtained by integrating the SPL spectrum:

$$
\mathrm{OASPL}=10 \log _{10} \int_{0}^{f_{\text {upper }}} 10^{0.1 \cdot \operatorname{SPL}(f)} d f
$$

where the upper integration limit is the highest frequency that can be resolved, in this case $15 \mathrm{kHz}$.

The time-dependent upward thrust $F_{z}$ produced by the flapping wings was measured by an ATI Nano17 Titanium load cell placed between the flapping-wing mechanism and its supporting post, Fig. 4. The range (and resolution) of the force measurable in the thrust direction is $28.2\left( \pm 5.15 \cdot 10^{-3}\right) \mathrm{N}$. The load cell is factory calibrated and the corresponding conversion factors are stored in the acquisition units used with it such that the values of the thrust obtained are already corrected. The load cell signal was sampled at $5 \mathrm{kHz}$ by the same acquisition system simultaneously recording the microphone signals. Each load-cell recording consists of $5 \cdot 10^{4}$ samples (thus the 
length of its timetrace is $10 \mathrm{~s}$ ). The reference upward thrust at each flapping frequency is the average of the corresponding time-varying values.

The supporting post also incorporates a Hall effect sensor for detecting the passage of a small neodymium magnet placed in the flapping-wing gearbox. This allows monitoring the flapping cycle and manually adjusting its frequency within $\pm 1 \mathrm{~Hz}$ of its nominal value. It also provides a reference during each flapping cycle for aligning the timetraces of the microphones and of the load cell which were acquired simultaneously albeit at different sampling frequencies. This enables the comparison and correlation of the acoustic and thrust signals.

\section{Experimental Results}

\section{A. Acoustic Measurements}

The environmental noise and the sound generated by the motor and the flapping-wing mechanism alone are evaluated before introducing the flapping wings and their sound.

The A-weighted OASPL measured inside the anechoic chamber with all the electric and electronic components turned on but without any movement of the flapping-wing model is about $27 \mathrm{dBA}$. This level is typical of a quiet bedroom and is lower than the level inside the average home.

All the wings were flapped by the same motor and flapping-wing mechanism. Measurements of its sound were recorded at flapping frequencies from 8 to $13 \mathrm{~Hz}$, i.e. from below to slightly above the reference $12 \mathrm{~Hz}$ flapping frequency of the FlowerFly. The lower flapping frequencies are representative of the take-off and approach/landing phases of flight whereas the flapping frequency of $13 \mathrm{~Hz}$ is representative of slight increases of thrust required to adjust the altitude and acceleration of the vehicle. Higher flapping frequencies have not been tested to avoid stressing and wearing the flapping mechanism so to maintain its performance as constant as possible during all the tests.

Figure 5 shows the sound characteristics measured at $0^{\circ}$ of the motor and flapping mechanism alone operating at $12 \mathrm{~Hz}$. Fig. 5 a) shows one second of the timetrace of the fluctuating pressure where the quasi-periodic nature of the pressure fluctuations can be distinguished. Figures 5 b) shows the corresponding A-weighted SPL spectrogram revealing that the sound of the motor and flapping mechanism comprises components at different audible

frequencies that are steady or quasi-steady in time. The details of the spectral content can be better observed in the A-weighted SPL spectrum of the whole timetrace, Fig. 5 c). The sound of the motor and flapping mechanism 
comprises several peaks two of which dominate at $4340 \mathrm{~Hz}$ and $7180 \mathrm{~Hz}$. The first peak closely matches the second harmonic of the frequency at which the teeth of the motor's pinion engage the teeth of the middle gear and its intensity is quite steady in time, Fig. 5 b). The origin of the peak at $7180 \mathrm{~Hz}$, which is broader and whose intensity is somewhat less steady, is less clear. We hypothesize it is the result of some friction between mutually sliding components but its precise identification would require specific additional investigation. Similar spectral characteristics have been obtained at the other angles around the motor and mechanism flapping at $12 \mathrm{~Hz}$.

Figure 6 a) shows one second of the timetrace measured at $0^{\circ}$ for wings A flapping at $12 \mathrm{~Hz}$. Strong pressure fluctuations at this flapping frequency can be seen that correlate to the clap-and-fling as discussed in the successive sub-section. Between these are smaller fluctuations corresponding to the clap-and-fling occurring at $\pm 90^{\circ}$. The corresponding spectrogram in Fig. 6 b) clearly reveals that strong components at different audible frequencies evenly repeat 12 times during one second. The spectrum of the whole timetrace in Fig. 6 c) shows that this wing type has a broad, high-intensity spectral distribution consistent with the rustling noise it produces during the flapping cycle. Small peaks emerge from this distribution of which the one at about $7200 \mathrm{~Hz}$ is steady in time and appears to be related to the sound of the flapping mechanism, see Figs. 5 b) and 5 c).

Figures 7 shows that wings A1 have characteristics similar to those of wings A in analogous flapping conditions but with lower pressure fluctuations and spectral amplitude. Compared to wings A and A1, the pressure fluctuations generated by wings $\mathrm{B}$ flapping at $12 \mathrm{~Hz}$ are much reduced as visible in Fig. 8 a). Accordingly, the spectral distributions in Figs. 8 b) and 8 c) show an amplitude reduction across most frequencies. As a result, the spectral components corresponding to the sound of the flapping mechanism emerge more prominently from the lower sound signature of this wing. Figure 9 summarizes the spectral findings discussed above by directly comparing the spectra of the flapping mechanism without and with the three types of flapping wing.

Similar spectral characteristics have been obtained for the cases above at other angles around the flapping-wing model. The corresponding OASPL values are presented in Fig. $10 \mathrm{c}$ ) whereas analogous values obtained at the flapping frequencies of 8 and $10 \mathrm{~Hz}$ are presented in Figs. 10 a) and $10 \mathrm{~b}$ ), respectively. As expected, the flapping wings are noisier than the mechanism alone and the overall sound increases with the flapping frequency for all the cases, consistent with the previous observations of $\mathrm{Lu}$ at al. [39] and of Deng et al. [40]. Also, the sound in all the cases is quite uniform around the flapping-wing model, in agreement with what can be experienced by a listener. This is also congruent with the double clap-and-fling of these flapping wings which creates similar sound sources 
every $90^{\circ}$. Wings B are clearly quieter than wings A1 which in turn are quieter than the standard wings A. This seems to validate that the flapping noise can be reduced by some tensing of the Mylar membrane so to reduce its flexing and twisting while maintaining a wing structure flexible enough for effective flapping.

\section{B. Upward Thrust Measurements and Performance Comparison}

Figure 11 shows the first $0.2 \mathrm{~s}$ of the timetrace of the pressure fluctuations of Fig. 6 a) together with the simultaneously recorded timetrace of the upward thrust measured by the load cell. The latter has been low-pass filtered at $200 \mathrm{~Hz}$ to remove irrelevant vibrations contaminating the significant features of the thrust. The timetraces are properly aligned relative to each other based on the simultaneously acquired timetrace of the Hall Effect sensor. A higher and a lower thrust peak are detected by the load cell every $0.0417 \mathrm{~s}$ consistent with the double clap-andfling at a flapping frequency of $12 \mathrm{~Hz}$. Strong pressure fluctuations trail every other thrust peak by about $0.014 \mathrm{~s}$ whereas smaller pressure fluctuations trail by the same amount the remaining thrust peaks. This strong correlation between the thrust and sound events suggests that the smaller pressure fluctuations are linked to the clap-and-fling occurring at $90^{\circ}$ relative to the angle of the clap-and-fling associated to the larger pressure fluctuations.

The average value of the upward thrust produced by the wings at each flapping frequency is plotted in Fig. 12 a). In all cases the thrust increases with the flapping frequency, as expected [38]. Wings B have larger thrust than wings A1 which in turn have larger thrust than the standard wings A. This may be the result of increasing the tension of the membrane of wings A1 and, especially, of wings B. The latter also have slightly larger wing surface which can also contribute to their thrust increase.

Since the OASPL values around the flapping wings are quite uniform, Fig. 10, their average can be used as the reference OASPL of the sound produced by the wings at each flapping frequency which is plotted in Fig. $12 \mathrm{~b}$ ). This figure also shows the values of the flapping mechanism alone (no wings) and synthesizes the results of Fig. 10, i.e. that the sound increases with the flapping frequency and that wings B are quieter than wings A1 themselves quieter than wings A.

Figure $12 \mathrm{c}$ ) plots the OASPL values produced by the wings at each flapping frequency as a function of the corresponding upward thrust. Since the FlowerFly weights about $20 \mathrm{~g}$, replacing its standard wings A with wings B could reduce its noise by about $9 \mathrm{dBA}$, i.e. the FlowerFly operating with wings B would be eight times quieter than operating with wings A. 
Figure $12 \mathrm{~d}$ ) presents the values of OASPL per unit upward thrust produced by the wings flapping at different frequencies and clearly shows that wings B are the best performers. Similar to what previously found for flapping wings of different types, the sound per unit thrust of each wing type decreases by increasing its flapping frequency [39]. This is a result of the thrust increasing more steeply with the flapping frequency than the corresponding sound. Thus, from a noise-reduction point of view, for a given wing type it is better to obtain the thrust required to fly a FW-MAV by designing wings and mechanisms that flap at higher frequency.

\section{Conclusion}

The main goal of this work was to explore if the perceived noise of the flapping wings of a MAV can be reduced by increasing the tension of their Mylar membrane. The reference wings are the standard type used in the FlowerFly MAV developed by the NUS Temasek Laboratories. These wings are quite similar to those used in the DelFly MAV develop by the MAVLab of TU Delft. Increasing the tension of the wings' Mylar membrane appears to both increase the upward thrust and decrease the noise produced by their flapping. Wings with curved stiffeners for tensing most of their membrane create lower noise per unit thrust than standard wings and could provide a $9 \mathrm{dBA}$ noise reduction if applied to a flapping-wing MAV. This suggests that curved stiffeners could be used in conjunction with thin, light, and strong materials more elastic than Mylar to further reduce the noise produced by flapping wings. Additionally, we found that the noise produced in a flapping cycle by four wings with double clap-and-fling is rather uniform around the wings, irrespective of the wing type or flapping frequency. The noise generated by such flapping wings increases with the flapping frequency albeit at a lower rate than their upward thrust. Thus the noise per unit thrust of a given wing type can be reduced by increasing its flapping frequency. These observations suggest that the flapping noise of a FW-MAV can be mitigated by designing wings with a slightly tensed membrane to reduce its flexing and twisting during the flapping cycle and that it is preferable to achieve the required upward thrust by sizing and operating such wings at higher rather than lower flapping frequencies.

\section{References}

[1] Fixed and flapping wing aerodynamics for micro air vehicle applications, edited by T. J. Mueller, Progress in astronautics and aeronautics, Vol. 195, AIAA, Reston, VA, 2001.

ISBN: 9781563475177 
[2] Shyy, W., Lian, Y., Tang, J., Viieru, D., and Liu, H., “Aerodynamics of low Reynolds number flyers,” Cambridge University Press, Cambridge, 2008.

ISBN: 9780521882781

[3] Pines, D. J., and Bohorquez, F., “Challenges Facing Future Micro-Air-Vehicle Development,” Journal of Aircraft, Vol. 43, No. 2, 2006, pp. 290-305.

doi: $10.2514 / 1.4922$

[4] Shyy, W., Berg, M., and Ljungqvist, D., "Flapping and flexible wings for biological and micro air vehicles," Progress in Aerospace Sciences, Vol. 35, No. 5, 1999, pp. 455-505. doi: 10.1016/S0376-0421(98)00016-5

[5] Platzer, M. F., Jones, K. D., Young, J., and Lai, J. C. S., "Flapping-Wing Aerodynamics: Progress and Challenges," AIAA Journal, Vol. 46, No. 9, 2008, pp. 2136-2149. doi: $10.2514 / 1.29263$

[6] Flying Insects and Robots, edited by D. Floreano, J.-C. Zufferey, M. V. Srinivasan, and C. Ellington, Springer-Verlag, Berlin Heidelberg, 2009. doi: 10.1007/978-3-540-89393-6_14

[7] de Croon, G. C. H. E., de Clerq, K. M. E., Ruijsink, R., Remes, B., and de Wagter, C., "Design, aerodynamics, and visionbased control of the DelFly," International Journal of Micro Air Vehicles, Vol. 1, No. 2, 2009, pp. $71-97$.

[8] de Croon G. C. H. E., Perçin M., Remes B. D. W., Ruijsink R., and de Wagter C., The DelFly: Design, Aerodynamics, and Artificial Intelligence of a Flapping Wing Robot, Springer, Dordrecht, Netherlands, 2016. doi: $10.1007 / 978-94-017-9208-0$

[9] Karásek, M., Muijres, F. T., De Wagter, C., Remes, B. D. W., de Croon, G. C. H. E., "A tailless aerial robotic flapper reveals that flies use torque coupling in rapid banked turns," Science, Vol. 361, No. 6407, 2018, pp. 1089-1094. doi: $10.1126 /$ science.aat 0350

[10] Finio, B. M., Eum, B., Oland, C., and Wood, R. J., “Asymmetric flapping for a robotic fly using a hybrid power-control actuator," Proceedings of the 2009 IEEE/RSJ International Conference on Intelligent Robots and Systems, St. Louis, MO, 2009, pp. 2755-2762.

doi: 10.1109/IROS.2009.5354424

[11] Ma, K. Y., Chirarattananon, P., Fuller, S. B., and Wood, R. J., "Controlled Flight of a Biologically Inspired, Insect-Scale Robot," Science, Vol. 340, 2013, pp. 603-607.

doi: 10.1126/science.1231806 
[12] Jafferis, N. T., Helbling, E. F., Karpelson, M., Wood, R. J., "Untethered flight of an insect-sized flapping-wing microscale aerial vehicle," Nature, Vol. 570, 2019, pp. 491-495. doi: 10.1038/s41586-019-1322-0

[13] Chapman, R. F., The Insects: Structure and Function, $4^{\text {th }}$ ed., Cambridge University Press, Cambridge, 1998. ISBN: 0521570484

[14] Sane, S. P., “The aerodynamics of insect flight,” The Journal of Experimental Biology, Vol. 206, 2003, pp. 4191-4208. doi: $10.1242 /$ jeb.00663

[15] Wang Z. J., "Dissecting Insect Flight," Annual Review of Fluid Mechanics, Vol. 37, 2005, pp. 183-210. doi: 10.1146/annurev.fluid.36.050802.121940

[16] Ellington, C. P., “The Aerodynamics of Hovering Insect Flight. I-VI,”, Philosophical Transactions of the Royal Society B, Biological Sciences, Vol. 305, No. 1122, 1984, pp. 1-181. doi: $10.1098 /$ rstb.1984.0049 to $10.1098 /$ rstb.1984.0054

[17] Ellington, C. P., van den Berg, C., Willmott, A. P., and Thomas, A. L. R., "Leading-edge vortices in insect flight," Nature, Vol. 384, 1996, pp. 626-630.

doi: $10.1038 / 384626 \mathrm{a} 0$

[18] Willmott, A. P., Ellington, C. P., and Thomas, A. L. R., "Flow visualization and unsteady aerodynamics in the flight of the hawkmoth, Manduca sexta," Philosophical Transactions of the Royal Society B, Biological Sciences, Vol. 352, No. 1351, 1997, pp. 303-316.

doi: $10.1098 /$ rstb.1997.0022

[19] van den Berg, C., and Ellington, C. P., “The vortex wake of 'hovering' model hawkmoth," Philosophical Transactions of the Royal Society B, Biological Sciences, Vol. 352, No. 1351, 1997, pp. 317-328. doi: $10.1098 /$ rstb.1997.0023

[20] van den Berg, C., and Ellington, C. P., "The three-dimensional leading-edge vortex of a 'hovering' model hawkmoth," Philosophical Transactions of the Royal Society B, Biological Sciences, Vol. 352, No. 1351, 1997, pp. 329-340. doi: 10.1098/rstb.1997.0024

[21] Dickinson, M. H., Lehmann, F.-O., and Sane, S. P., "Wing Rotation and the Aerodynamic Basis of Insect Flight," Science, Vol. 284, No. 5422, 1999, pp. 1954-1960. doi: $10.1126 /$ science. 284.5422 .1954

[22] Birch, J. M., and Dickinson, M. H., "Spanwise flow and the attachment of the leading-edge vortex on insect wings," Nature, Vol. 412, 2001, pp. 729-733. doi: $10.1038 / 35089071$ 
[23] Ansari, S. A., Phillips, N., Stabler, G., Wilkins, P. C., Żibkowski, R., and Knowles, K., "Experimental investigation of some aspects of insect-like flapping wing aerodynamics for application to micro air vehicles," Experiment in Fluids, Vol. 46, 2009, pp. 777-798.

doi: 10.1007/s00348-009-0661-2

[24] Wang, Z. J., “Two Dimensional Mechanism for Insect Hovering,” Physical Review Letters, Vol. 85, No. 10, 2000, pp. 22162219.

doi: 10.1103/PhysRevLett.85.2216

[25] Sun, M., and Tang, J., "Unsteady aerodynamic force generation by a model fruit fly wing in flapping motion," The Journal of Experimental Biology, Vol. 205, 2002, pp. 55-70.

[26] Sun, M., and Tang, J., "Lift and power requirements of hovering flight in Drosophila virilis," The Journal of Experimental Biology, Vol. 205, 2002, pp. 2413-2427.

[27] Miller, L. A., and Peskin, C. S., “A computational fluid dynamics of 'clap and fling' in the smallest insects," The Journal of Experimental Biology, Vol. 208, 2005, pp. 195-212.

doi: $10.1242 /$ jeb.01376

[28] Sun, M., and Yu, X., "Aerodynamic Force Generation in Hovering Flight in a Tiny Insect," AIAA Journal, Vol. 44, No. 7, 2006, pp. 1532-1540.

doi: $10.2514 / 1.17356$

[29] Aono, H., Liang, F. and Liu, H., "Near- and far-field aerodynamics in insect hovering flight: an integrated computational study," The Journal of Experimental Biology, Vol. 211, 2008, pp. 239-257.

doi: $10.1242 /$ jeb.008649

[30] Sueur, J., Tuck, E. J. and Robert, D., "Sound radiation around a flying fly,” Journal of the Acoustic Society of America, Vol. 118, No. 1, 2005, pp. 530-538.

doi: 10.1121/1.1932227

[31] Bae, Y., and Moon, Y. J., "Aerodynamic sound generation of flapping wing," The Journal of the Acoustical Society of America, Vol. 124, No. 1, 2008, pp. 72-81.

doi: $10.1121 / 1.2932340$

[32] Inada, Y., Aono, H., Liu, H., and Aoyama, T., "Numerical Analysis of Sound Generation of Insect Flapping Wings," Theoretical and Applied Mechanics Japan, Vol. 57, 2009, pp. 437-447. doi: $10.11345 /$ nctam. 57.437

[33] Geng, B., Xue, Q., Zheng, X., Liu, G., Ren, Y., and Dong, H., "The effect of wing flexibility on sound generation of flapping wings," Bioinspiration \& Biomimetics, Vol. 13, No. 1, 2018, 016010. 
doi: 10.1088/1748-3190/aa8447

[34] G eng, B., Zheng, X., Xue, Q., Liu, G., and Dong, H., "A Numerical Study of the Sound and Force Production of Flexible Insect Wings," Fluids, Vol. 3, No. 4, 2018, 87.

doi: 10.3390/fluids3040087

[35] Insect sound and communication - Physiology, Behavior, Ecology, and Evolution, edited by S. Drosopoulos, and M. F. Claridge, CRC press, Taylor and Francis Group, Boca Raton, Florida, 2005.

doi: $10.1201 / 9781420039337$

[36] Swartz, S. M., Groves, M. S., Kim, H. D., and Walsh, W. R., "Mechanical properties of bat wing membrane skin,” Journal of Zoology, Vol. 239, No. 2, 1996, pp. 357-378.

doi: 10.1111/j.1469-7998.1996.tb05455.x

[37] Swartz, S. M., Bishop, K. L., and Aguirre I. M-F., "Dynamic complexity of wing form in bats: implications for flight performance," Functional and Evolutionary Ecology of Bats, edited by Z. Akbar, G. F., McCracken, and T. H. Kunz, Oxford University Press, Oxford, 2006, pp. 110-130.

ISBN: 978-0195154726

[38] Nguyen, Q. V., Chan, W. L. and Debiasi, M., "Experimental Investigation of Wing Flexibility on Force Generation of a Hovering Flapping Wing Micro Air Vehicle with Double Wing Clap-and-Fling Effects,” International Journal of Micro Air Vehicles, Vol. 9, No. 3, 2017, pp. 187-197.

doi: $10.1177 / 1756829317695565$

[39] Lu, Z., Debiasi, M., Nguyen, Q. V., and Chan, W. L., “Bioinspired Low-Noise Wing Design for a Two-Winged FlappingWing Micro Air Vehicle,” AIAA Journal, Vol. 56, No. 12, 2018, pp. 4697-4705.

doi: 10.2514/1.J056293

[40] Deng, S., Wang, J., Zhang, Y., and Cai, H., "Experimental Investigation of Aerodynamic and Acoustic Characteristics of a Flapping Wing Micro-Air-Vehicle," AIAA Paper 2019-2619, May 2019.

doi: 10.2514/6.2019-2619 

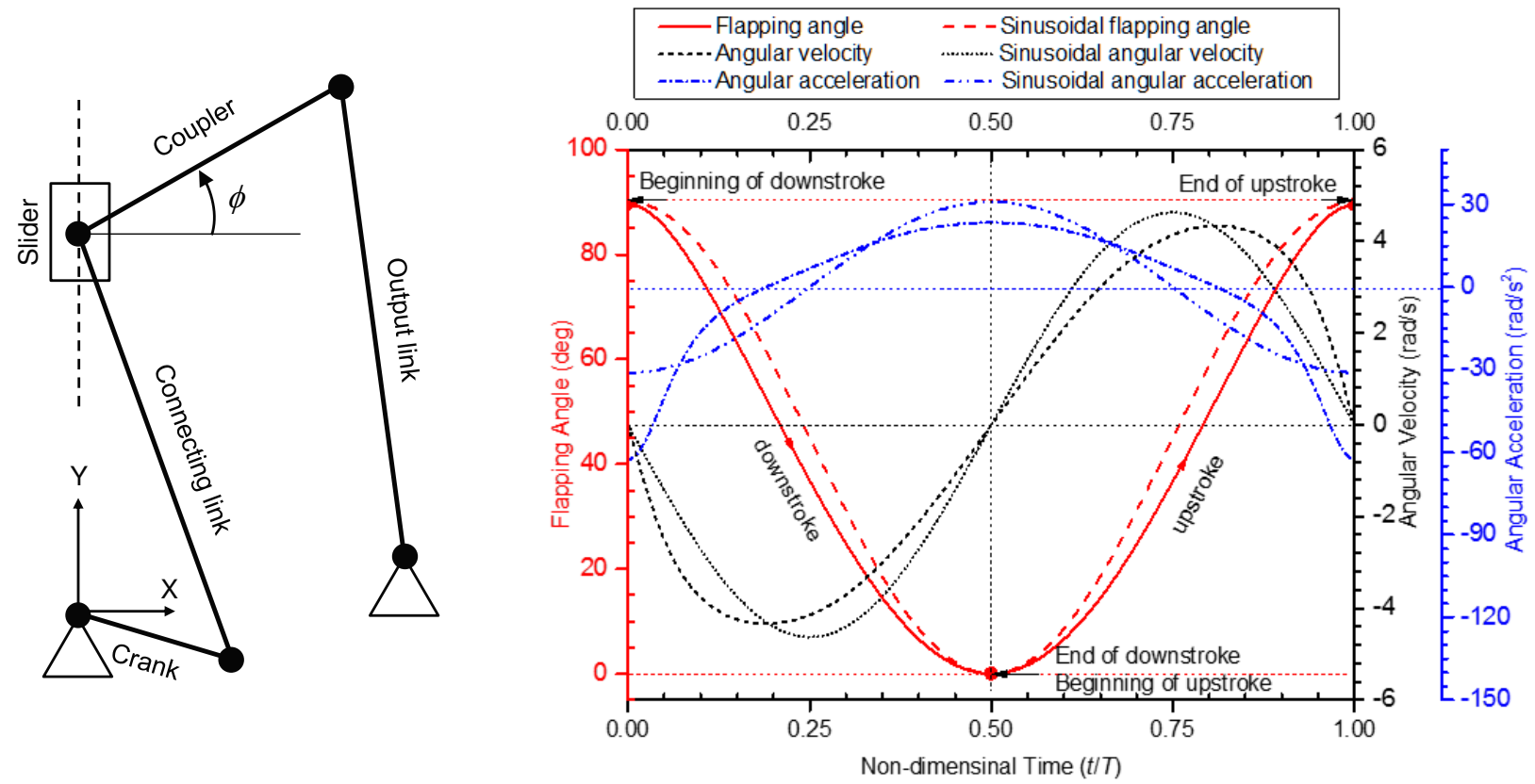

Fig. 1 Schematic of the crank-slider and linkages of the FlowerFly flapping mechanisms (left), and flapping angle $\phi$, of the output link (right). Sinusoidal functions are for reference. 

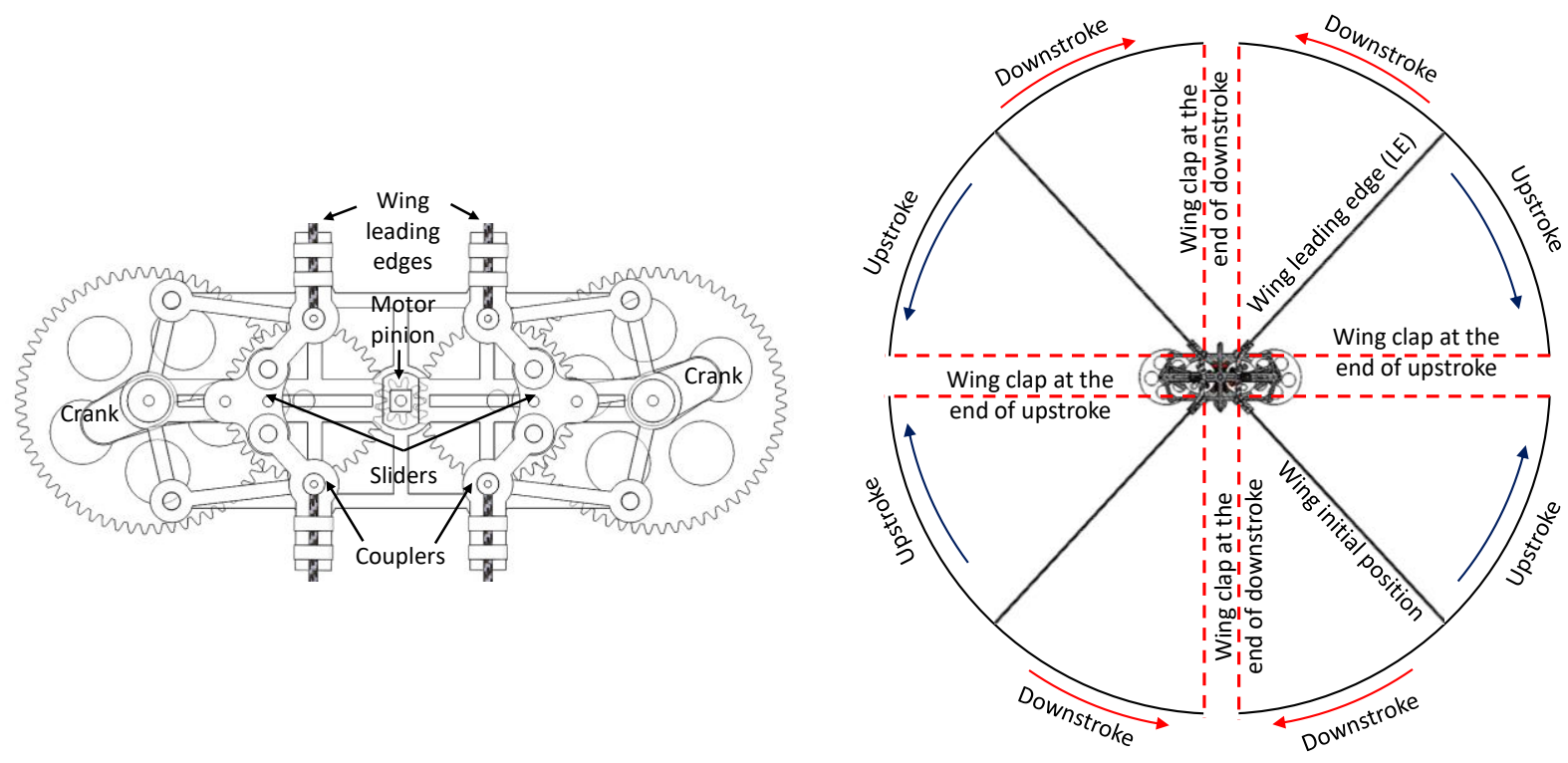

Fig. 2 Design of gear box for double clap-and-fling effects (left), and flapping sequence (right): end of upstroke or beginning of downstroke - first clap, end of downstroke or beginning of upstroke - second clap. 

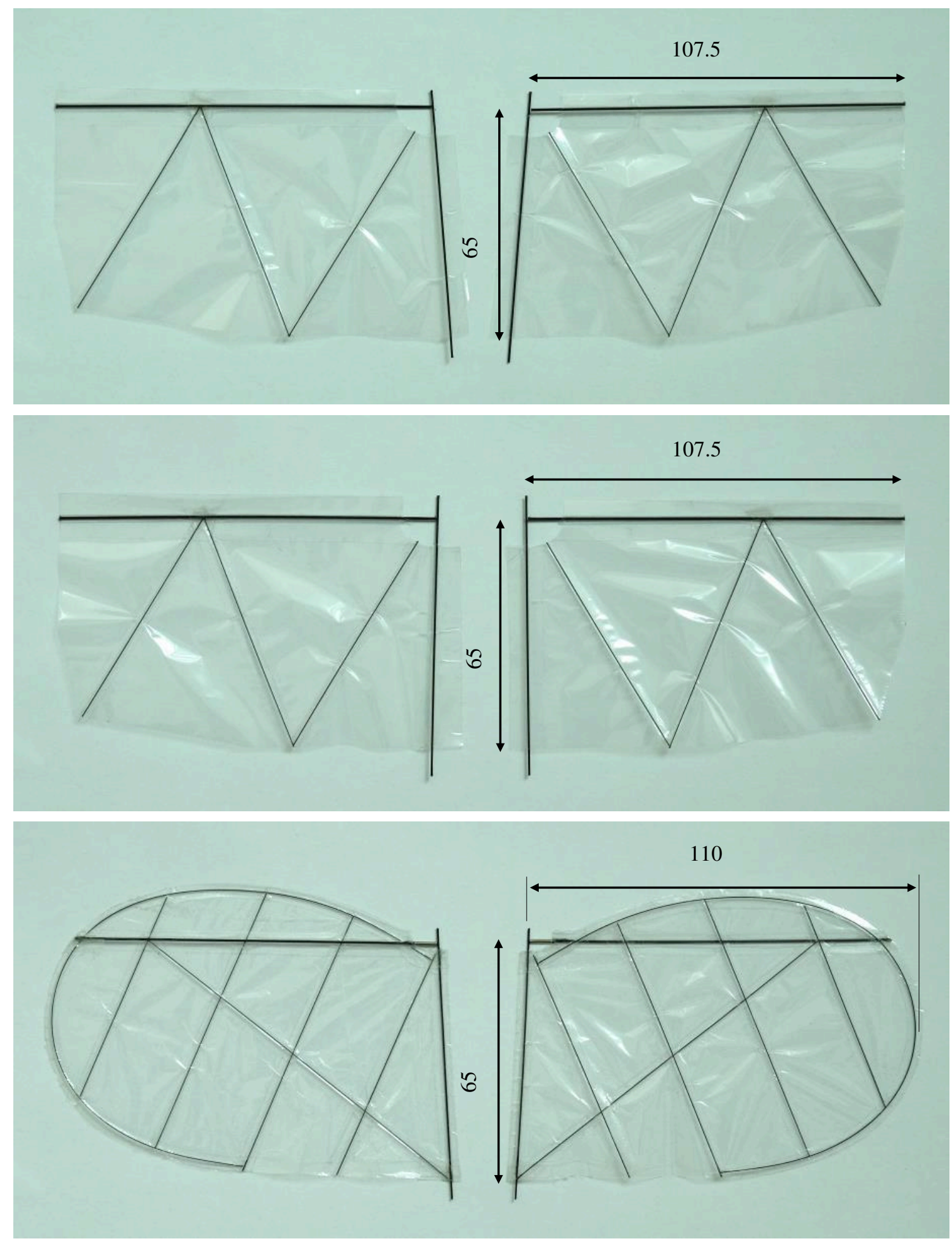

Fig. 3 Tested wings: standard type A (top), type A1 (middle), and type B (bottom). Dimensions are in mm. 

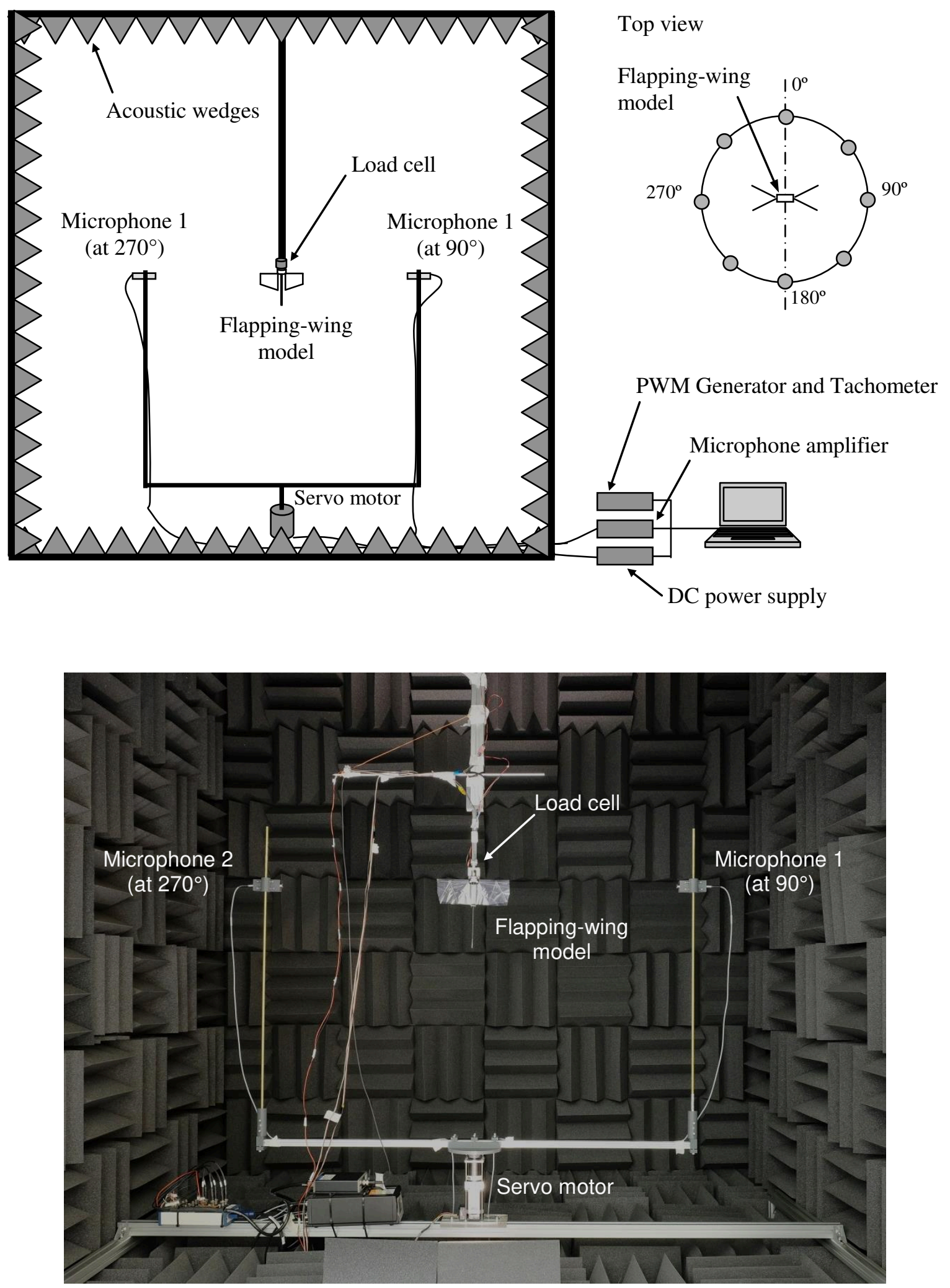

Fig. 4 Schematic drawing (top) and picture (bottom) of the experimental setup in the anechoic chamber. 
a)

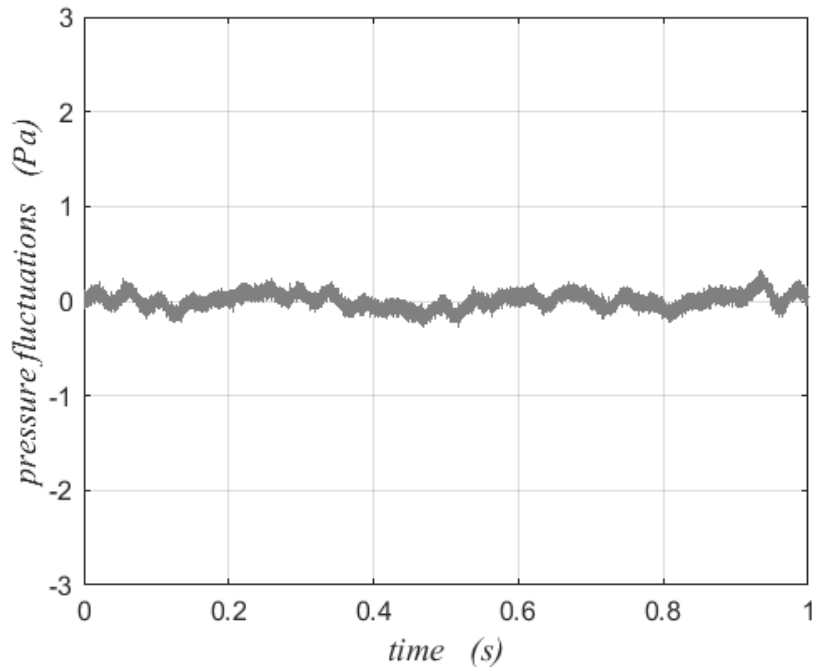

b)

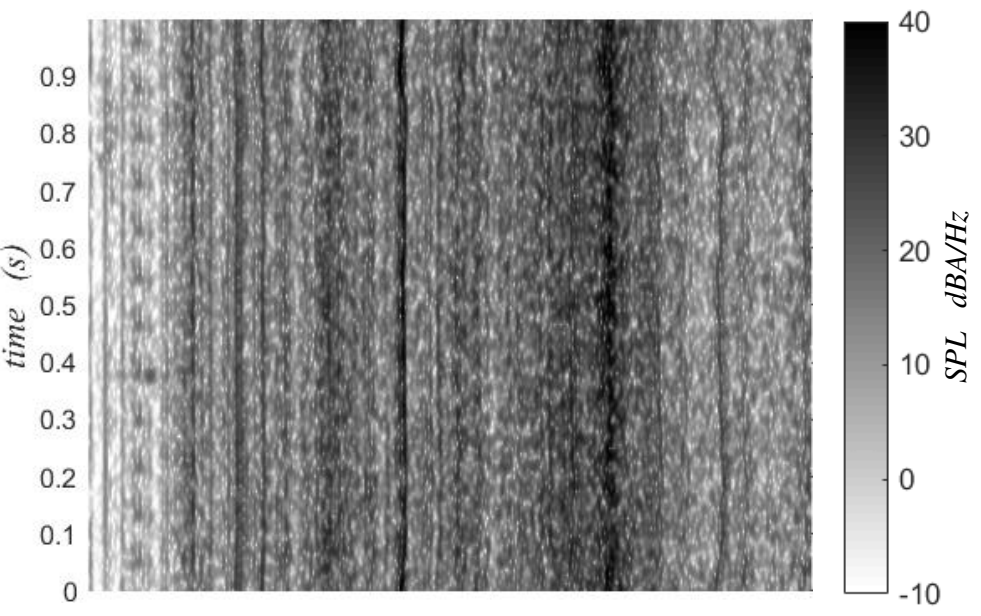

c)

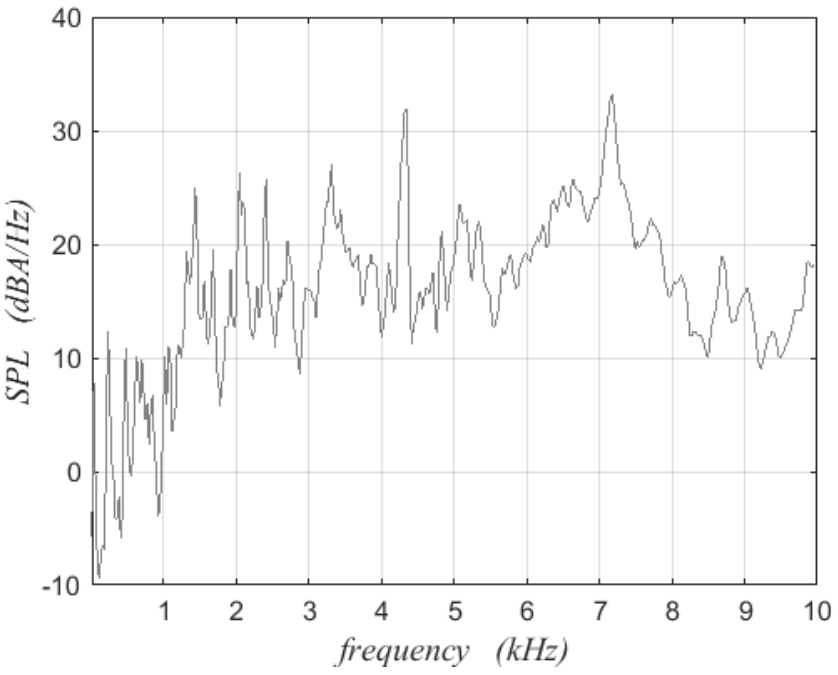

Fig. 5 Sound measured at $0^{\circ}$ of the motor and flapping mechanism alone operating at 12 Hz: a) timetrace of the pressure fluctuations; b) A-weighted SPL spectrogram; c) A-weighted SPL spectrum. 
a)

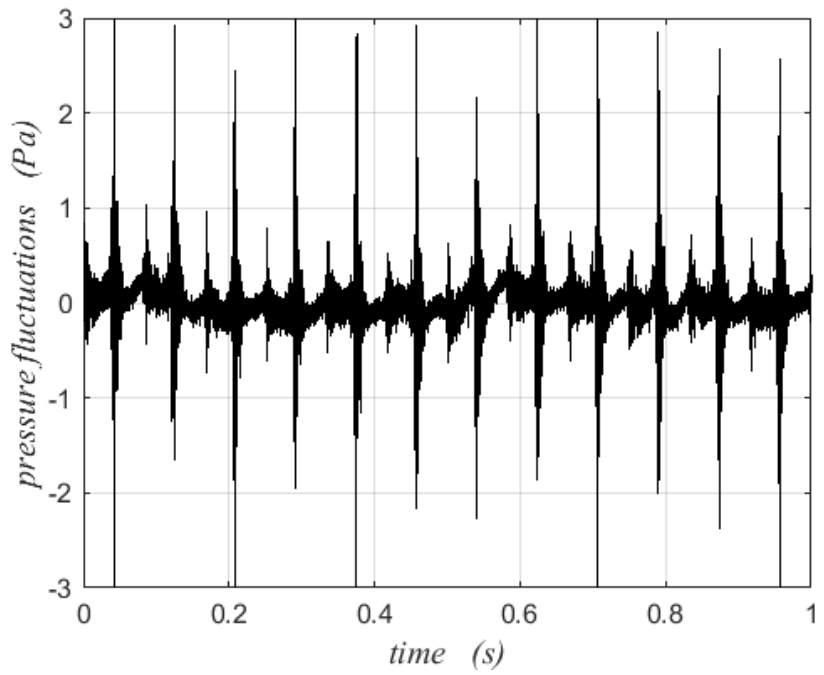

b)
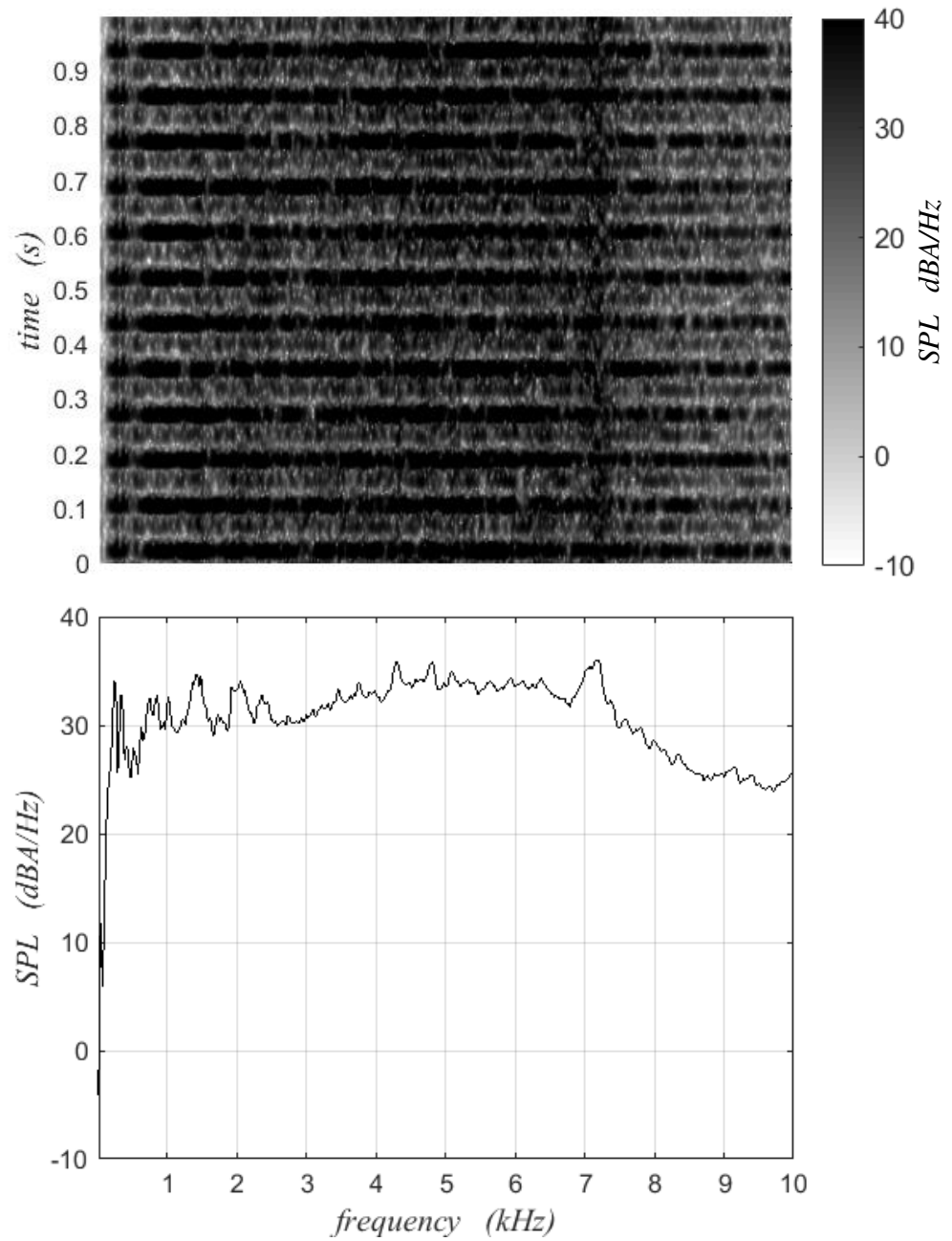

Fig. 6 Sound measured at $0^{\circ}$ of the A wings flapping at $12 \mathrm{~Hz}$ : a) timetrace of the pressure fluctuations; b) Aweighted SPL spectrogram; c) A-weighted SPL spectrum. 
a)

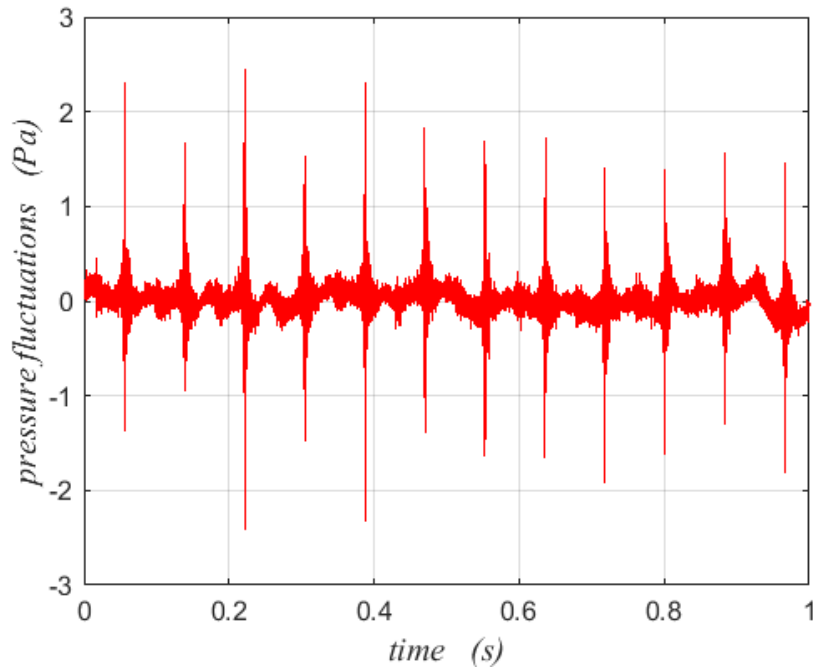

b)

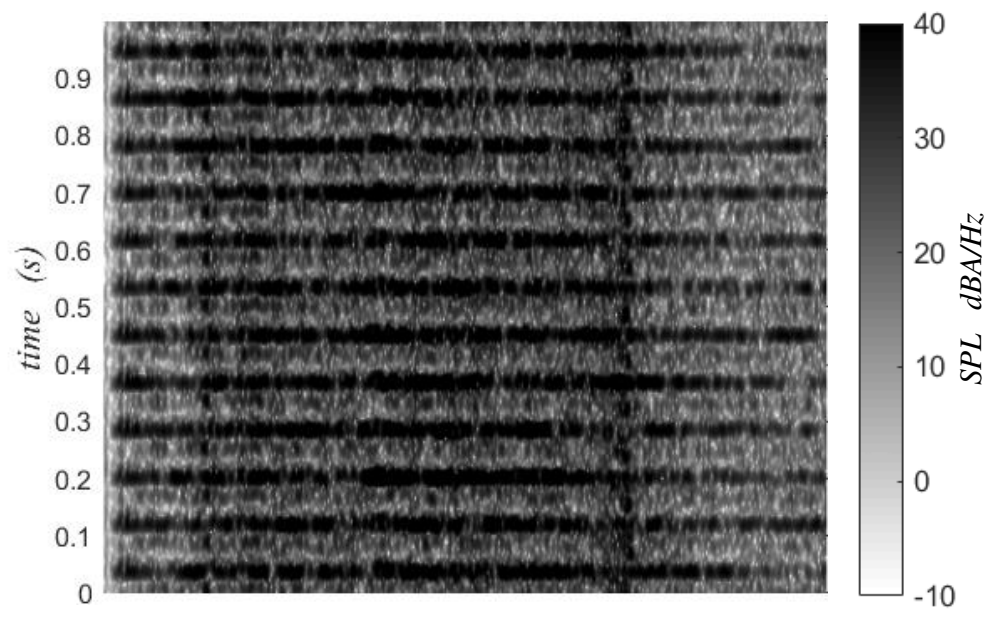

c)

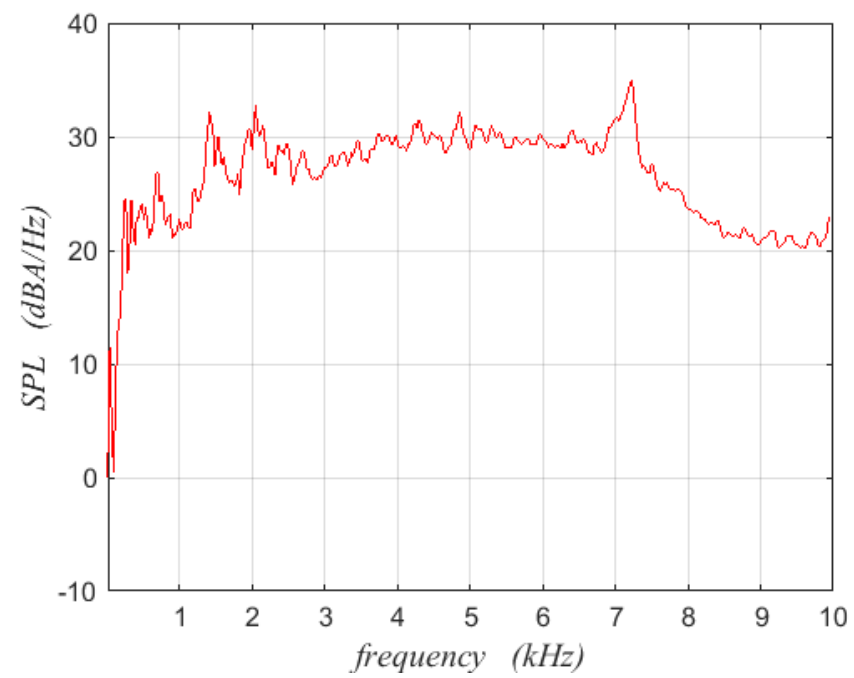

Fig. 7 Sound measured at $0^{\circ}$ of the A1 wings flapping at $12 \mathrm{~Hz}$ : a) timetrace of the pressure fluctuations; b) Aweighted SPL spectrogram; c) A-weighted SPL spectrum. 
a)

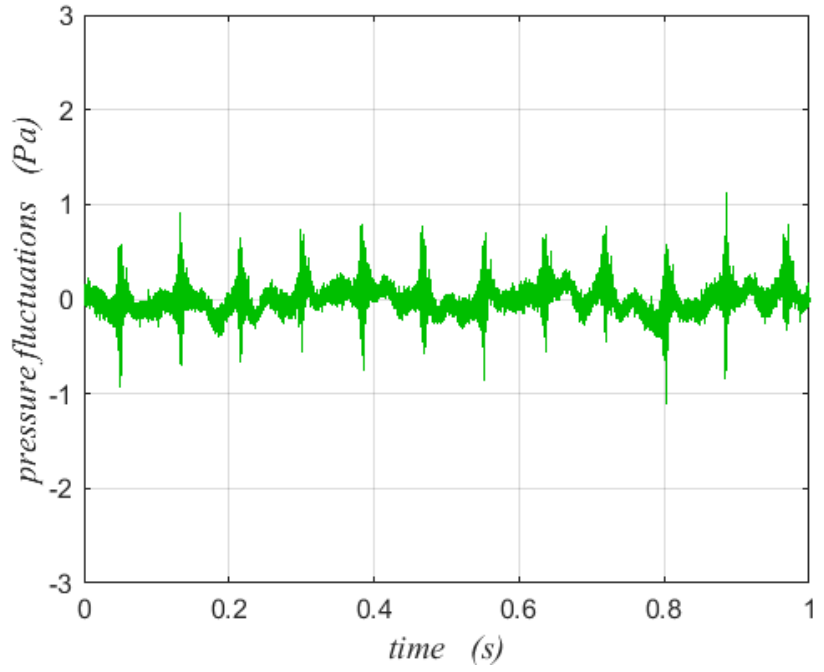

b)

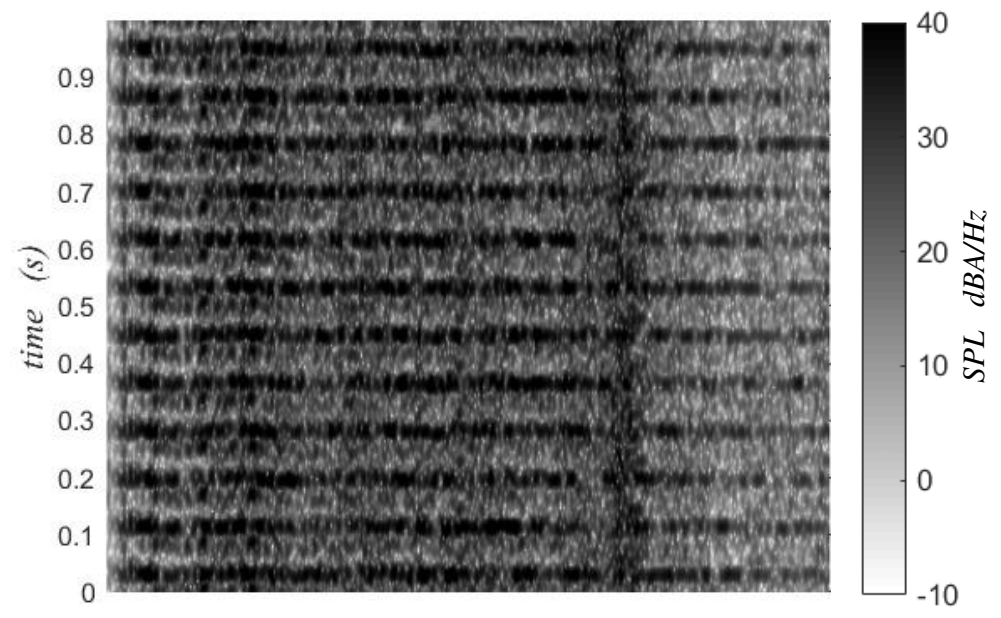

c)

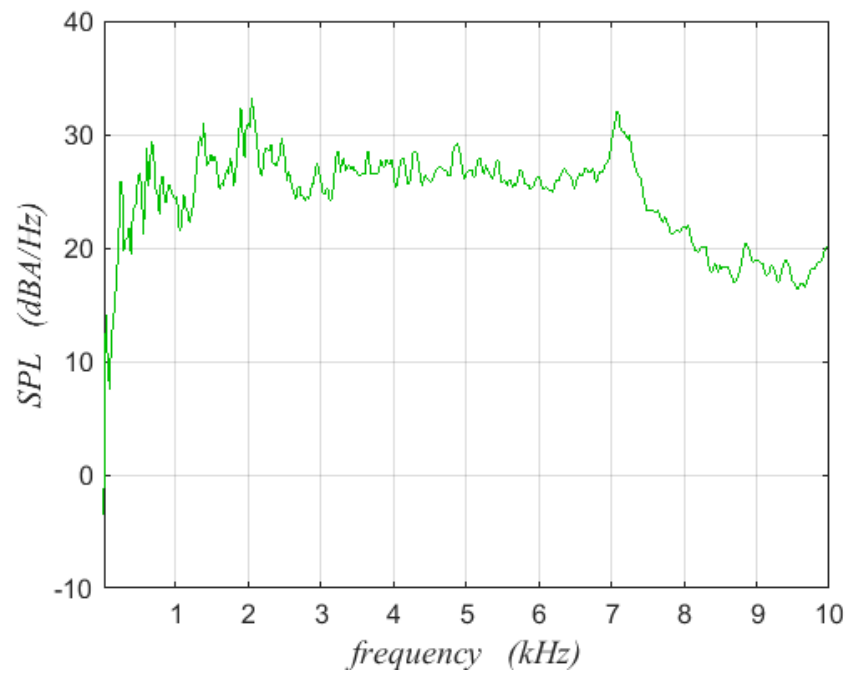

Fig. 8 Sound measured at $0^{\circ}$ of the $B$ wings flapping at $12 \mathrm{~Hz}$ : a) timetrace of the pressure fluctuations; b) Aweighted SPL spectrogram; c) A-weighted SPL spectrum. 


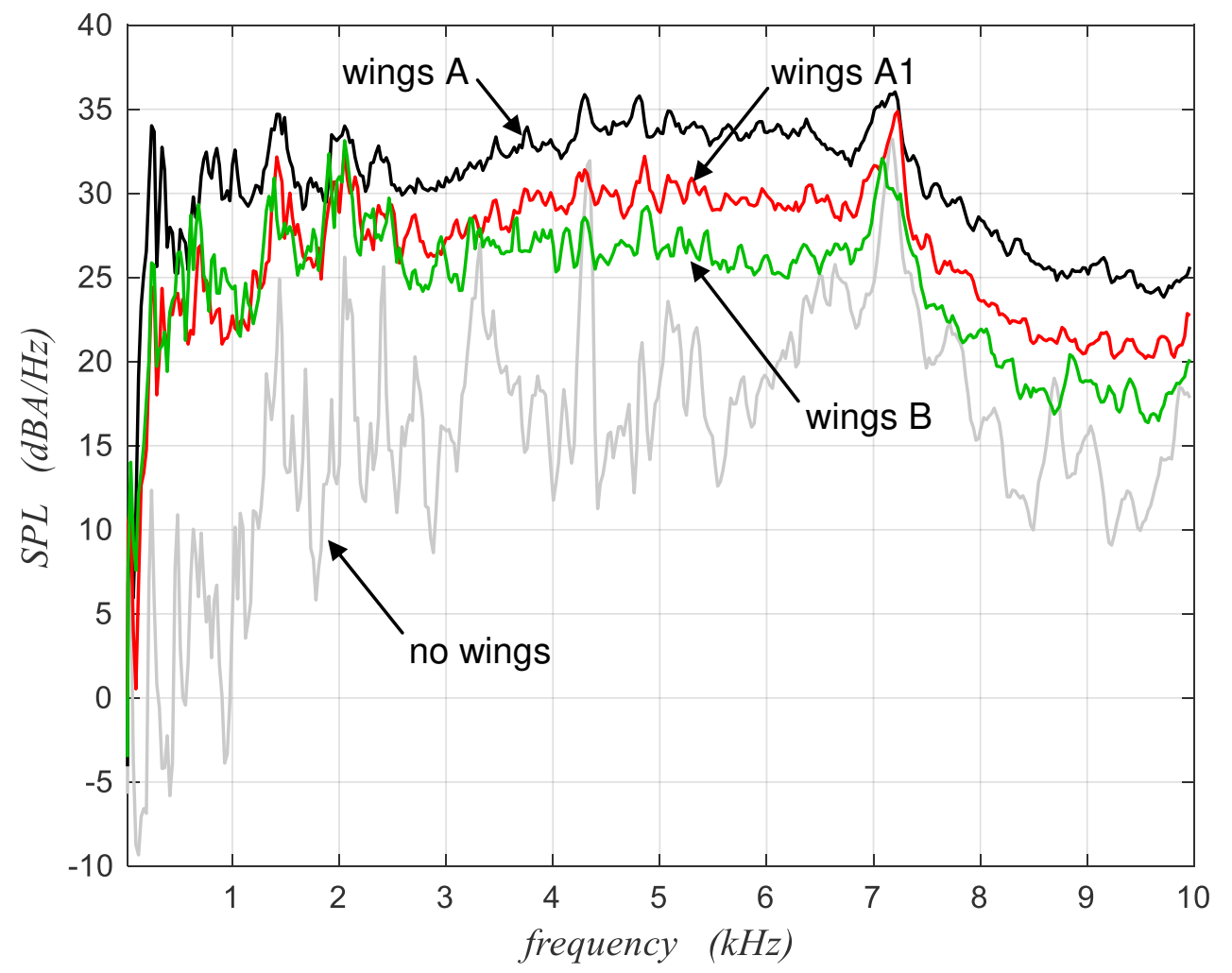

Fig. 9 A-weighted SPL spectra of the sound measured at $0^{\circ}$ of the different types of wing flapping at $12 \mathrm{~Hz}$. 


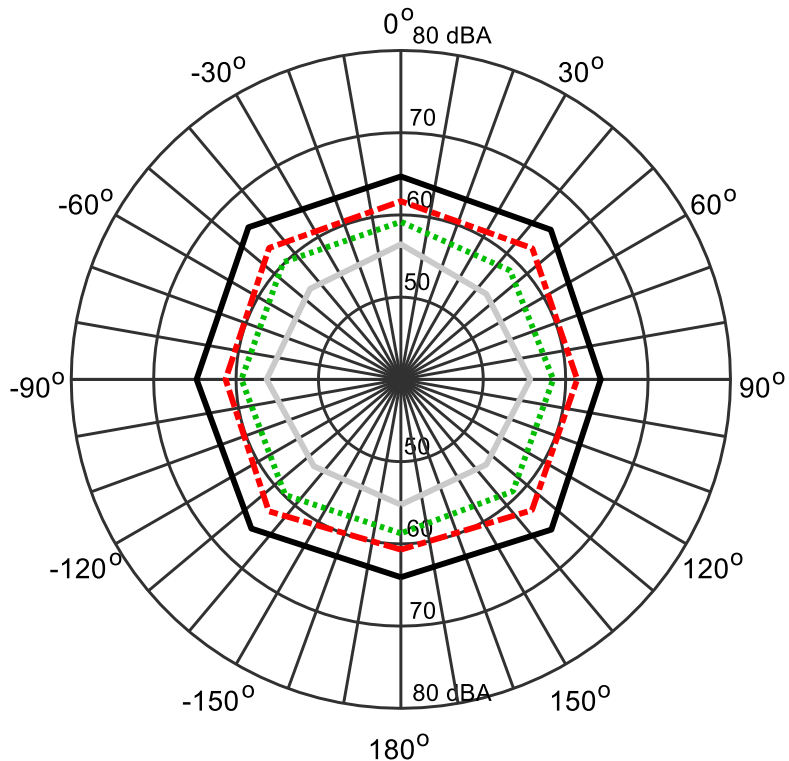

a)

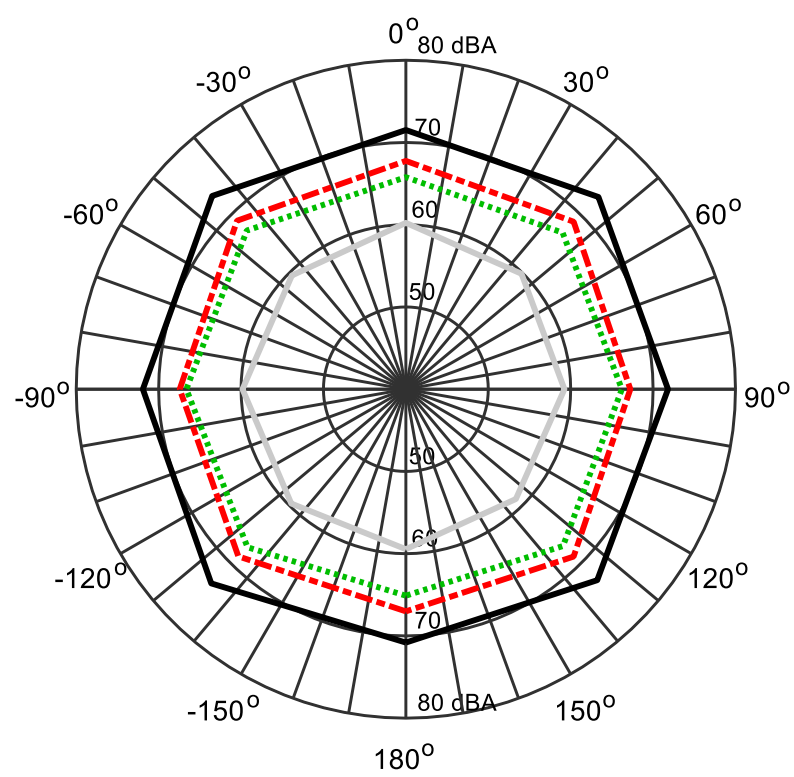

c)

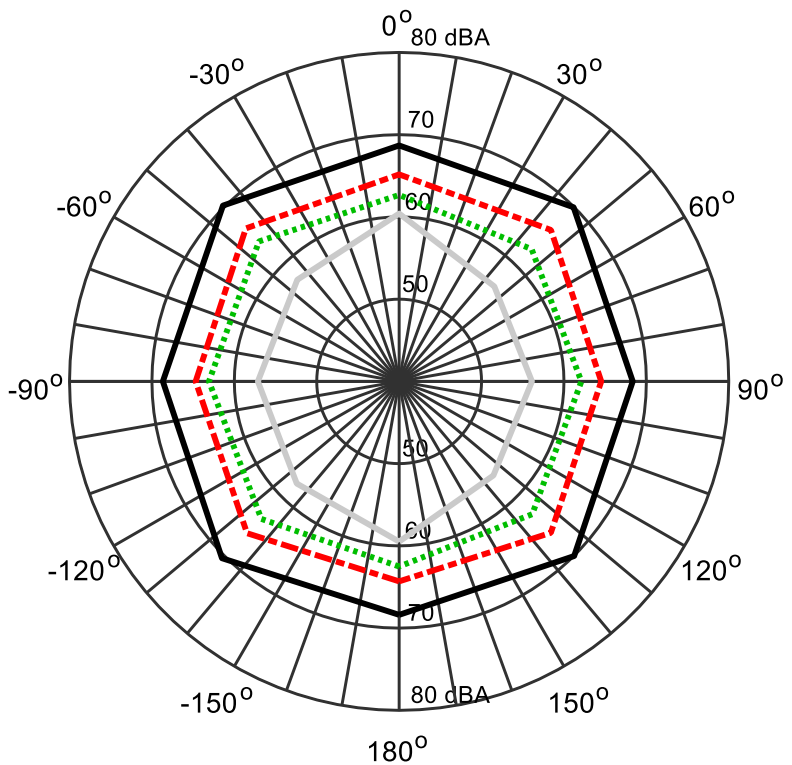

b)

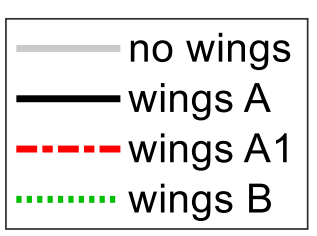

Fig. 10 A-weighted OASPL values measured around the wings flapping at: a) $8 \mathrm{~Hz}$; b) $10 \mathrm{~Hz}$;) $12 \mathrm{~Hz}$. 


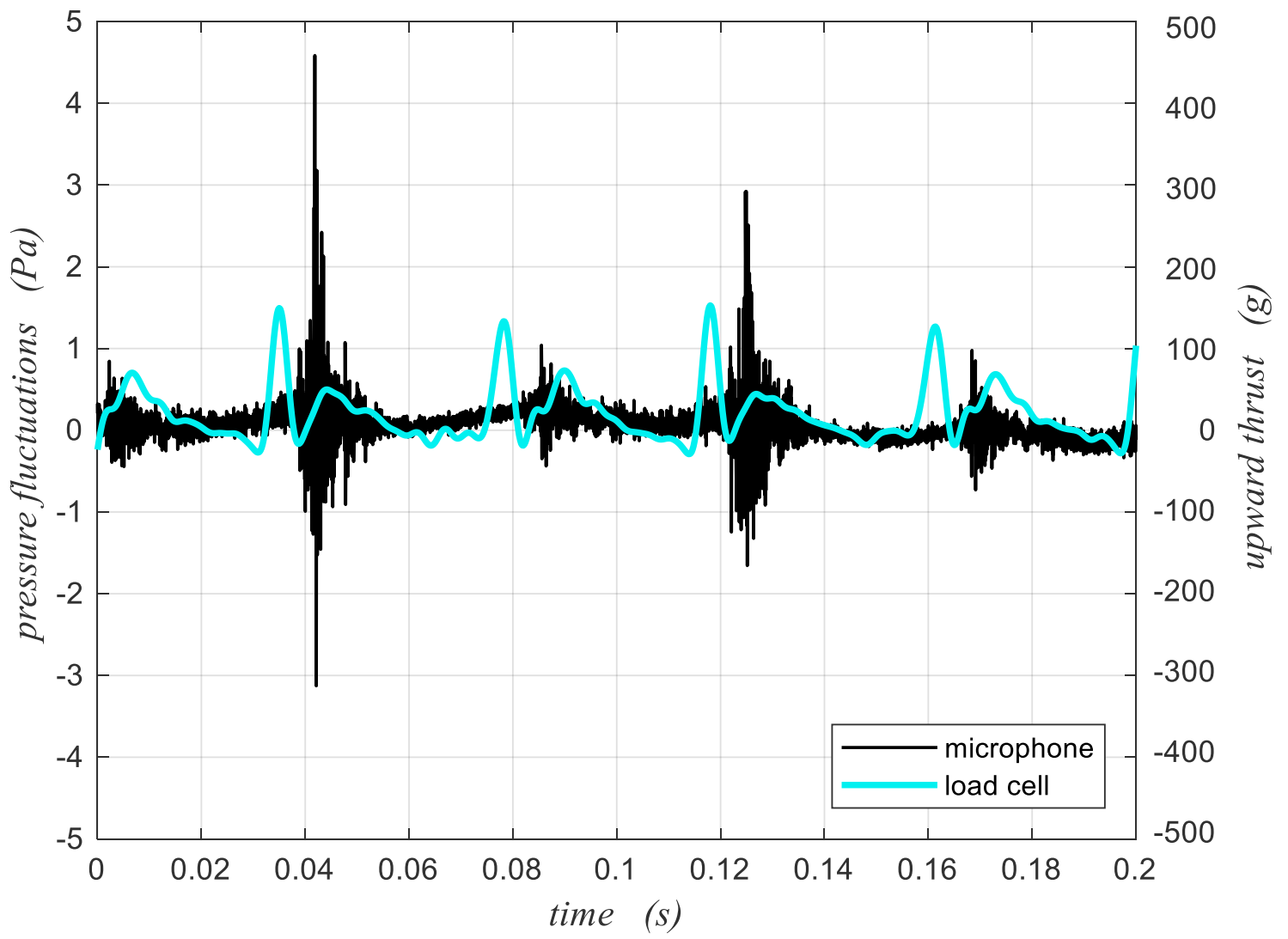

Fig. 11 Timetraces of the pressure fluctuations measured at $0^{\circ}$ and of the upward thrust of wings A flapping at $12 \mathrm{~Hz}$. 

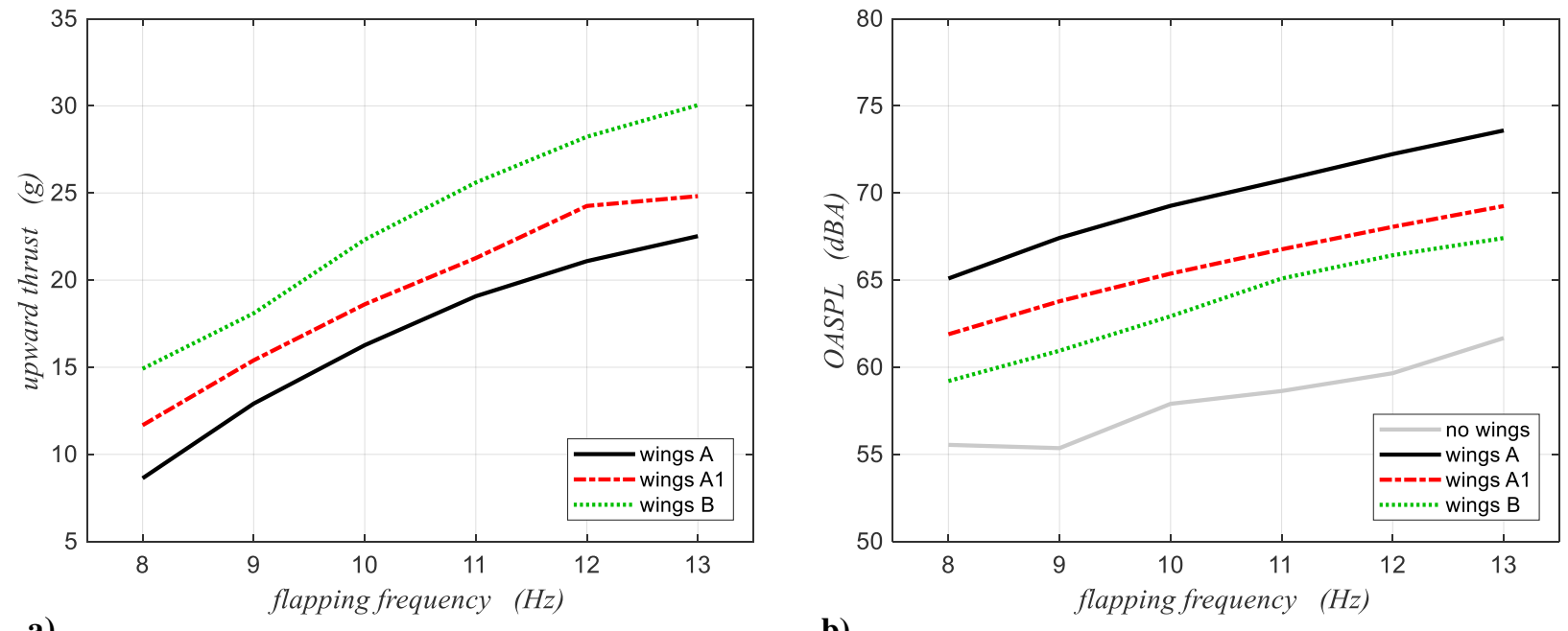

a)

b)
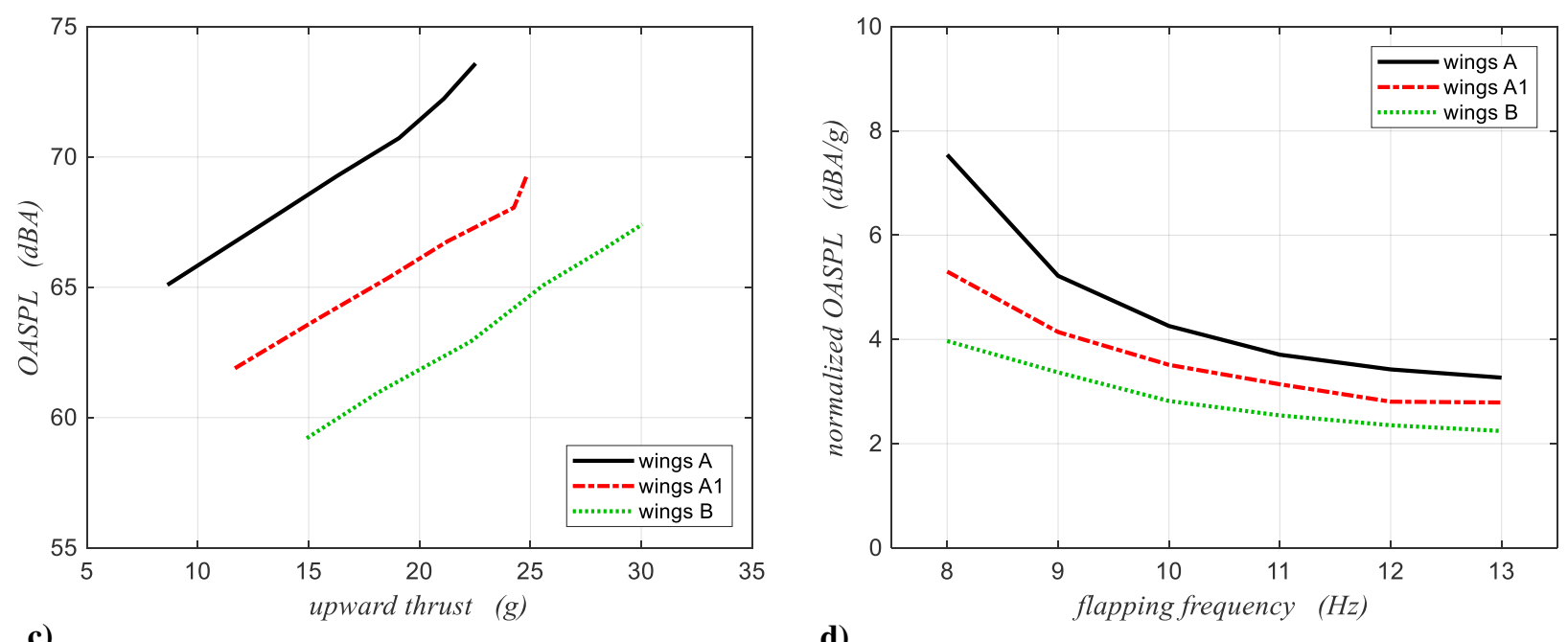

d)

Fig. 12 Characteristics of the flapping wings: a) upward thrust as a function of the flapping frequency; b) Aweighted OASPL values as a function of the flapping frequency; c) A-weighted OASPL values as a function of the upward thrust; d) A-weighted OASPL values per unit upward thrust as a function of the flapping frequency. 